\title{
Amygdalar sclerosis: preoperative indicators and outcome after temporal lobectomy
}

\author{
Laurie A Miller, Richard S McLachlan, M Susan Bouwer, Lawrence P Hudson, \\ David G Munoz
}

\begin{abstract}
Isolated amygdalar sclerosis (AS) in the presence of an intact hippocampus has been described in a subset of patients who have undergone a temporal lobectomy for the relief of seizures. Clinical observation suggested that these patients might be distinguishable, before and after operation, from those with typical mesial temporal sclerosis, which implies combined amygdalar and hippocampal sclerosis (AHS). From a three year series, all 11 patients classified as having AS were included in this study. These patients were compared with a group of 20 randomly chosen patients with AHS. The groups were found to be well matched in duration of ongoing seizures, full scale IQ, and duration of follow up (mean 19 months). Compared with patients with AHS, patients in the AS group were less likely to have had a seizure in early childhood, a variety of auras, EEG abnormalities localised to one temporal lobe, or an abnormal MRI before operation. They also performed better on preoperative memory tests. At follow up, patients in the AS group were less likely to be seizure free and more likely to have a deterioration in memory after undergoing anterior temporal lobectomy, including part of the hippocampus. The results show that there are preoperative indicators of mesial temporal pathology that are also of prognostic importance given the differences in outcome between the two pathological groups.
\end{abstract}

$(\Im$ Neurol Neurosurg Psychiatry 1994;57:1099-1105)

Department of Clinical Neurological Sciences L A Miller R S McLachlan M S Bouwer

Department of Pathology, University of Western Ontario, London, Ontario, Canada

L P Hudson

D G Munoz

Correspondence to:

Dr Richard McLachlan,

Department of Neurology,

University Hospital, 339

Wiversity Hospital, 339

Ontario, N6A 5A5 Canada.

Received 9 July 1993 and in final revised form

18 April 1994.

Accepted 27 April 1994
There was also evidence of a moderate to severe memory impairment in patients with AHS, and only mild memory deficits in those with isolated AS.

The presence of mesial temporal (or "hippocampal") sclerosis in the surgically resected lobe is associated with a reduced incidence of postoperative seizures. ${ }^{23}$ The relation of AS to seizure outcome after temporal lobectomy has not been explored. Clinical observation (by LAM and RSM), however, has suggested that patients with AS have poorer postoperative seizure control than patients with AHS.

Furthermore, the role of the hippocampus in memory function is now well established. Given that patients in the AS group underwent temporal lobe removals that included seemingly normal hippocampal tissue, postoperative decreases in memory for patients in this group might be expected. If indeed outcome for seizure control or memory function differs for these patients, then it becomes important to identify preoperative measures that are sensitive to the extent of mesial temporal pathology because, in the future, these measures could help to determine prognosis. Therefore, as well as outcome, several possible preoperative indicators were used to compare patients with AS and those with AHS.

\section{Methods}

SUBJECTS

A consecutive series of 113 patients (age $\geqslant 13$ years) who had a temporal lobectomy carried out at University Hospital for the relief of intractable seizures between January 1989 and December 1991 were considered for the present study. Surgery was carried out when $(a)$ a focal seizure onset was shown, $(b)$ the risks of surgery were low, and $(c)$ the patient was psychologically prepared for surgery. Patients were assessed by standard EEG telemetry with scalp or when necessary, implanted subdural electrodes. Neuropsychological assessments and MRI or CT were also done. Patients were excluded from the study if $(a)$ an inadequate sample of tissue had been provided for pathology for either establishing or ruling out the presence of hippocampal or amygdalar sclerosis, (b) a non-sclerotic lesion was found in the mesial temporal region (for example, hippocampal tumour), or (c) an additional discrete lesion was identified outside the mesial area. Forty two cases were excluded for these reasons.

In 11 of the 71 remaining patients, AS was found. For comparison, 20 patients with AHS 
were selected at random from a total of 60 possible cases. These patients had a loss of neurons and gliosis involving the amygdala as well as the $\mathrm{CA}_{1}$ and $\mathrm{CA}_{3}$ hippocampal sectors, with partial sparing of $\mathrm{CA}_{2}$ (as described by Falconer and others $\left.{ }^{2-6}\right)$. Patients were not divided according to side of lesion because of the limited number of subjects in the AS group.

\section{POSTOPERATIVE SEIZURE OUTCOME}

At least one year of postoperative neurological follow up was achieved in all cases. Outcome was determined by a neurologist (RAM) blind to the pathological results based on information obtained from the patient and at least one relative either at the last follow up appointment or by phone interviews with patients who had not had recent postoperative assessments. Each patient was rated as to whether or not they had $(a)$ been seizure free (excluding auras) since surgery, $(b)>90 \%$ reduction in seizures, (c) $50-90 \%$ reduction in seizures, or (d) $<50 \%$ reduction in seizures. The presence or absence of postoperative grand mal seizures was also evaluated.

\section{ELECTROENCEPHALOGRAPHY}

Preoperative EEGs with and without telemetry were reviewed blind to the pathology by RSM and the location of background slowing, interictal spikes, and seizures were categorised as exclusively unitemporal, wide field (unitemporal with prominent extratemporal propagation), or independent bitemporal. The presence of generalised spike wave activity was also noted. Electroencephalography (EcoG) was carried out with surface electrodes over the convexity of the temporal lobe and inferior frontal lobe with a single depth probe inserted into the mesial temporal region $2-3 \mathrm{~cm}$ from the temporal tip.

\section{NEUROPSYCHOLOGY}

The educational level achieved by each patient as well as their preoperative and postoperative WAIS-R IQ scores were recorded. Wherever possible, preoperative and postoperative performance on a number of memory measures was evaluated. Nine patients in the AHS and seven patients in the AS group had been retested one or more years after their operation. Group mean scores on the memory measures were used to examine changes in performance after operation. Cut off scores were also used to rate each patient's performance as either impaired or unimpaired. The cut off scores were determined based on the results of a previous study. ${ }^{6}$ The

Table 1 Demographic data of subjects

\begin{tabular}{|c|c|c|c|c|c|c|}
\hline \multirow[b]{2}{*}{ Group } & \multicolumn{2}{|c|}{ Sex } & \multicolumn{2}{|c|}{ Side of excision } & \multirow{2}{*}{$\begin{array}{l}\text { Age } \\
(y \text { (range) })\end{array}$} & \multirow{2}{*}{$\begin{array}{l}\text { Seizure } \\
\text { duration } \\
(y \text { (range) })\end{array}$} \\
\hline & $M$ & $F$ & Left & Right & & \\
\hline $\begin{array}{l}\text { Amygdalar and } \\
\text { hippocampal sclerosis }\end{array}$ & 9 & 11 & 13 & 7 & $31(15-51)$ & $18(6-35)$ \\
\hline Amygdalar sclerosis & 5 & 6 & 6 & 5 & $30(13-47)$ & $13(3-31)$ \\
\hline
\end{tabular}

tests considered and the scores representing the lowest unimpaired level for each were: WMS paired word associate learning score 14; WMS paired word associate per cent delayed recall score 80; WMS logical passages per cent delayed recall score 60; WMS (immediate) visual reproduction score 8; Rey complex figure per cent delayed recall score 50; Warrington recognition memory test: words (age adjusted, scaled score) 7; and Warrington's recognition memory test: faces (age adjusted, scaled score) 7 .

\section{NEUROLOGICAL HISTORY}

A blind review of patient records was made for the following preoperative clinical variables: age at initial seizure, age at onset of ongoing complex partial seizures, duration of ongoing complex partial seizures, incidence of preoperative grand mal seizures, and aura type(s).

\section{NEURORADIOLOGY}

Preoperative cranial MRI examinations with blinded interpretation were performed for all patients. Temporal lobe atrophy and increased T2 signal in the hippocampal region have been found to be the most helpful indicators for diagnosing hippocampal sclerosis before operation ${ }^{7-12}$; however, our previous study $^{1}$ showed that these same abnormalities are also suggested in a subset of the patients with isolated AS. Therefore, in the present investigation, the reviewer was asked to identify only the cases in which there were definite indications of either temporal lobe atrophy or increased T2 signal.

\section{STATISTICAL ANALYSES}

$\chi^{2}$ tests, $t$ tests, and repeated measure analyses of variance were used to evaluate group differences. For the $\chi^{2}$ tests, if any estimated cell size fell below 5, Yates' correction was employed. Results for which a p value $<0.05$ was achieved are reported as significant; trends are reported where $0 \cdot 1>p \geqslant 0 \cdot 05$.

\section{Results}

\section{DEMOGRAPHIC VARIABLES}

Mean duration of follow up was 19 months for the 31 patients in the study and there was no significant difference between the groups in duration of follow up. Table 1 gives demographic information on the patient groups. $\chi^{2}$ tests showed no significant differences for sex or side of lesion distribution.

Furthermore, $t$ tests showed no group differences in mean age or mean years of seizure duration.

\section{POSTOPERATIVE SEIZURE OUTCOME}

Patients in the AS group had a greater tendency to experience postoperative seizures than did those in the AHS group ( $p=0.08)$. Twelve patients with AHS and three with AS were seizure free, four in each group had more than a $90 \%$ reduction in seizures, two from each group had $50-90 \%$ seizure reduction, and two in each group failed to achieve $50 \%$ 
reduction in seizures. There was a trend for patients in the AHS group to be completely seizure free after operation (60\%), which was not the case of those without hippocampal pathology (27\% seizure free). For half of the patients who had postoperative seizures, these were rare events (mean less than two seizures per year), usually occurring in association with reduction in medication. Four patients in each group had ongoing, regularly occurring seizures postoperatively.

Groups did not differ in postoperative grand mal seizure tendency. Most (about $60 \%$ ) of the patients who experienced seizures after operation had at least one grand mal. This was equally true for both of the patient groups. There was only one patient (AHS group) who experienced postoperative grand mal seizures, who had not had at least one preoperative grand mal.

\section{EEGS}

Of the 31 patients, 13 had telemetry with placement of subdural electrodes, four in the AS group and in nine with AHS. There were no obvious differences in interictal spike frequency between patients in the AS and AHS groups. Table 2 shows the distribution of electrographic abnormalities. Significant differences in the location of background slowing $\left(\chi^{2}=8.56, \mathrm{p}<0.02\right)$ and focal spikes $\left(\chi^{2}\right.$ $=9.75, \mathrm{p}<0.05)$ were found in AS compared with AHS, these abnormalities being more widespread in the first. None of the spikes in patients who had AS were localised to a single temporal lobe. Similarly, seizure onset was somewhat less likely to be confined to one temporal lobe in patients with AS $55 \%$ of patients) compared with AHS $(90 \%$ of patients). Seizure propagation also seemed to be more widespread in patients with AS compared with the AHS group. Only three patients had generalised spike wave activity, one with AS and two with AHS. No differences in location or frequency of spike activity recorded by ECoG were noted in the two groups. Spike activity predominated from the depth electrode compared with convexity electrodes in $73 \%$ of patients with AS compared with $55 \%$ with AHS. This possibly relates to variable non-stereotaxic placement

Table 2 Preoperative electroencephalographic findings

\begin{tabular}{lll}
\hline & $\begin{array}{l}\text { Amygdalar and } \\
\text { hippocampal sclerosis (n) }\end{array}$ & $\begin{array}{l}\text { Amygdalar } \\
\text { sclerosis }(n)\end{array}$ \\
\hline Background slowing & & \\
$\quad$ Unitemporal & 15 & 3 \\
Bitemporal & 2 & 5 \\
None & 3 & 3 \\
Interictal spikes & & \\
$\quad$ Unitemporal & 9 & 0 \\
Widefield & 5 & 5 \\
Bitemporal & 6 & 4 \\
None & 0 & 2 \\
Seizure onset: & & \\
Unitemporal & 18 & 6 \\
Widefield & 0 & 2 \\
Bitemporal & 1 & 2 \\
None recorded & 1 & 1 \\
\hline${ }^{\star}$ < 0.05 & &
\end{tabular}

of the depth electrode that may have been in or near the amygdala more often than in the hippocampus.

\section{NEUROPSYCHOLOGY}

The patient groups were found to be well matched in terms of academic achievement; a $t$ test yielded no significant between group difference in mean level of education. For these subjects, the average duration of education was 11.9 years. Similarly, $t$ tests yielded no significant between group differences for either preoperative (mean $=90$ ) or postoperative full scale IQ (mean $=95$ ).

For patients who were tested both before and after operation, repeated measure group $x$ time of testing analyses were used to compare performance at the two testing times. These gave significant interactions for full scale IQ $(F=7.35, \mathrm{p}<0.05)$ and per cent recall of the Rey complex figure $(F=17 \cdot 5$, p $<0.01$ ). As shown in fig 1 , for full scale IQ, the AHS group had a significant improvement after surgery $(t=3.04, \mathrm{p}<0.05)$, but there was no significant change in the mean IQ score of the AS group. Figure 2 illustrates the results for the Rey complex figure. The AHS group again showed significant improvements after surgery $(t=3.27, \mathrm{p}<0.05)$, and, in this case, the performance of the AS group deteriorates $(t=3.16, \mathrm{p}<0.05)$. None of the other interactions involving the memory measures reached significance, but for several (WMS visual reproduction, Warrington words, and Warrington faces), a similar pattern was seen, in that the scores for the AS group decreased after operation and those of the AHS either increased slightly, or showed no significant change. For the WMS paired word associate learning measure, there was a significant main effect of time of testing $(F=5 \cdot 1, p<0.05)$. Both groups showed a decline in the ability to learn the associates when tested one to two years after surgery.

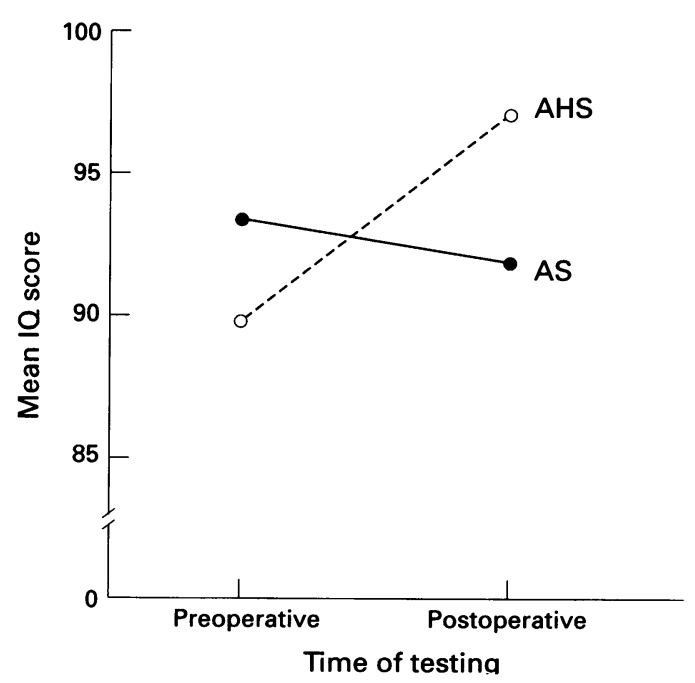

Figure 1 Full scale IQ. There was a significant group $\times$ time testing interaction as the full scale IO of the AHS group improved significantly after operation whereas that of the $A S$ group did not alter. 


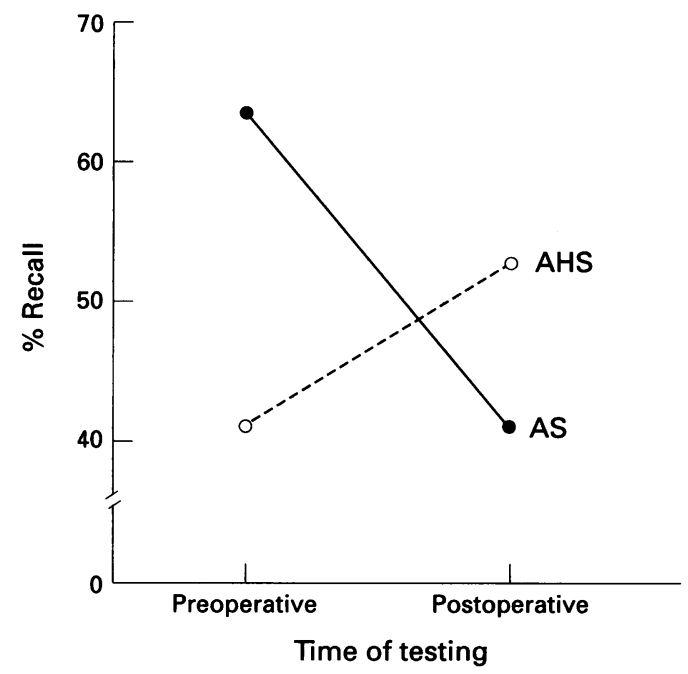

Figure 2 Percentage recall of the Rey complex figure. There was a significant group $\times$ time of testing interaction as the mean score of the AHS group improved after operation but that of the AS group decreased significantly.

Cut off scores were used to determine the percentage of subjects in each group who were impaired on each measure. As can be seen in table 3, the measures that best distinguished the AHS group from the AS group preoperatively were Rey figure per cent delayed recall $\left(\chi^{2}=4.04, p<0.05\right)$ and WMS visual reproduction ( $\chi^{2}$ [with Yates' correction] $=3.24, p=0.07$ ). Again, overall, the failure rate of the AS group tended to increase postoperatively, whereas that of the AHS group tended either to fall or to remain virtually unchanged.

Given that the group that showed postoperative memory deterioration was also the one that had the least effective seizure control, it seemed possible that the presence of postoperative seizures might be influencing memory performance. When patients were divided according to seizure outcome (seizure free $v$ not seizure free), however, there were no significant differences in postoperative memory performance. Thus the differences in neuropsychological outcome between the AS and AHS groups does not seem to be secondary to differences in postoperative seizure control.
Table 3 Failure rate on memory tests (percentage of group below cut off)

\begin{tabular}{|c|c|c|c|c|}
\hline \multirow[b]{2}{*}{ Memory measure } & \multicolumn{2}{|c|}{$\begin{array}{l}\text { Amygdalar and } \\
\text { hippocampal sclerosis }\end{array}$} & \multicolumn{2}{|c|}{ Amygdalar sclerosis } \\
\hline & $\begin{array}{l}\text { Preoperative } \\
(\%)\end{array}$ & $\begin{array}{l}\text { Postoperative } \\
(\%)\end{array}$ & $\begin{array}{l}\text { Preoperative } \\
(\%)\end{array}$ & $\begin{array}{l}\text { Postoperative } \\
(\%)\end{array}$ \\
\hline Rey figure delayed recall & 65 & 33 & 27 & 67 \\
\hline WMS visual reproduction & 50 & 22 & 9 & 14 \\
\hline Warrington recognition & & & & \\
\hline memory: words & 42 & 33 & 11 & 33 \\
\hline Warrington recognition & & 44 & & \\
\hline $\begin{array}{l}\text { memory: faces } \\
\text { WMS log passages }\end{array}$ & 37 & 44 & 40 & 67 \\
\hline$\%$ delayed recall & 43 & 38 & 40 & 43 \\
\hline $\begin{array}{l}\text { WMS paired word learning } \\
\text { WMS paired word }\end{array}$ & 47 & 56 & 36 & 71 \\
\hline$\%$ delayed recall & 22 & 22 & 36 & 14 \\
\hline
\end{tabular}

Correlation tests were used to determine whether there was a significant relation between medication level and performance on the memory measures. No significant correlations were found either before or after operation. Also, the two patient groups did not differ either before or after operation in mean dosage of anticonvulsant medication or percentage of the group taking more than one drug (about $67 \%$ preoperatively and $17 \%$ postoperatively for both groups). These results indicate that differences on memory tasks between the two groups cannot be attributed to differences in medication levels.

\section{NEUROLOGICAL HISTORY \\ Seizures}

A $\chi^{2}$ test revealed a significant difference $\left(\chi^{2}=\right.$ $15.9, \mathrm{p}<0.001)$ between the groups in the percentage of patients who had an initial seizure (febrile or afebrile) at or before 5 years of age. Whereas $80 \%(16 / 20)$ of patients with AHS had had a seizure before the age of 5 years ( 14 before 2 years), this had not happened in any of the patients in the AS group. The earliest seizures occurred in the AS group at the age of 7 years (this was the case for two patients). For the 15 patients with AHS who had had a seizure early in life, the initial seizure in 11 of these cases was known to have occurred in association with a fever, four had seizures associated with a cause other than fever (for example, head trauma), and one had had an initial seizure of unknown cause. By contrast, a $t$ test showed no difference with respect to age at onset of ongoing complex partial seizures. The mean age was 15 years.

A $\chi^{2}$ test showed a trend $(p=0.07)$ for the two groups to differ in the number of patients who experienced preoperative grand mal seizures; whereas all patients in the AS group had had at least one grand mal seizure before surgery, seven of 20 patients in the AHS group had never had one.

Types and numbers of auras

Auras were categorised as cephalic or epigastric sensation, sensation of smell or taste, autonomic, headache, fear, or other experiental phenomena (for example, déjà vu, hallucinations). In the AS group, four patients had no aura, six had one aura type, and one had two or more aura types, compared with the AHS group in which the numbers were two, nine, and nine respectively. Thus nearly half (nine of 20) in the AHS group had two or more aura types, whereas this was true for only one of the patients in the AS group. Table 4 presents the percentage of patients in each group that reported experiencing the different types of auras preoperatively. Of interest is the relatively high percentage of patients with AS having no aura (36\%). Although this was not analysed statistically because of the small sample sizes, the aura types that seemed to distinguish the groups best were smell or taste sensations and fear. A higher proportion of patients in the AHS group had these types of auras than patients in the AS group. An equal percentage of patients in the two groups 
Table 4 Type of aura

\begin{tabular}{lll}
\hline Aura & $\begin{array}{l}\text { Amygdalar and } \\
\text { hippocampal } \\
\text { sclerosis (\%) }\end{array}$ & $\begin{array}{l}\text { Amygdalar } \\
\text { sclerosis } \\
(\%)\end{array}$ \\
\hline None & 10 & 36 \\
Cephalic/epigastric & 45 & 45 \\
Smell/taste & 30 & 0 \\
Autonomic & 20 & 9 \\
Headache & 5 & 0 \\
Fear & 40 & 9 \\
Other experiential & 25 & 18 \\
\hline
\end{tabular}

reported experiencing a cephalic or epigastric sensation before a seizure, which has long been considered a classic sign of temporal lobe epilepsy. ${ }^{13} 14$

\section{Neuroradiology}

A clear difference between the groups $\left(\chi^{2}=\right.$ $18.2, \mathrm{p}<0.001)$ was found in the preoperative MRI results. The presence of definite temporal lobe atrophy or an increased T2 signal suggestive of mesial temporal sclerosis in the hippocampal region of the operated hemisphere was identified in 16 of 20 patients with hippocampal pathology and in none of the patients in the AS group. It should be noted, however, that for two of the 11 patients in the AS group, MRI did show an abnormality in the amygdaloid region, which preoperatively had been thought to be a tumour.

\section{Discussion}

Isolated sclerosis of the amygdala was found in about $10 \%$ of patients who underwent temporal lobectomy for the relief of intractable seizures. This contrasts with the much higher percentage (about $53 \%$ ) of patients who were found to have typical mesial temporal sclerosis, involving both the amygdala and the hippocampus and no other disorder. Thus it seems, not unexpectedly, that AHS is a much more prevalent condition than isolated AS in patients with temporal lobe epilepsy. It is possible, however, that the present sample was biased in this direction, because some of the preoperative factors found to be associated with AHS (for example the presence of an MRI abnormality) are ones that would have increased the likelihood of a temporal lobectomy being carried out. On the other hand, there is no evidence that the severity or intractability of epilepsy, the fundamental determinants for surgical consideration, are any different in the two pathological conditions, AHS and AS.

The importance of identifying AS preoperatively is clear; patients with this pathological condition are less likely to become seizure free ( $27 \%$ compared with $60 \%$ with AHS). The reason surgery is less successful in controlling seizures in the AS group is unknown but this finding is consistent with previous ones that absence of pathological evidence of mesial temporal sclerosis (hippocampal sclerosis) in the resected tissue suggests a poor prognosis. Furthermore, after undergoing temporal lobe excisions that include the anterior hippocampus, patients with AS are more likely to show memory deterioration than are those with more extensive mesial temporal pathology. In selected patients, an excision that includes all of the amygdala but spares the hippocampus has a similar rate of success in controlling seizures as a more extensive excision that includes part of the body of the hippocampus. ${ }^{15}{ }^{16}$ It has been postulated ${ }^{16}$ that an excision limited to the amygdalar region might spare memory function, compared with a more extensive mesial removal. Such a surgical procedure would seem especially indicated for patients with AS.

This study has produced some factors that can be used before operation to predict whether the hippocampus is involved in the underlying pathological condition. As has been found in the past, clinical history of a seizure in early childhood ${ }^{1-5131718}$ was an extremely good predictor of mesial temporal sclerosis involving the hippocampus; this history was not indicated for any of the patients in the AS group. In the present study, the presence of temporal lobe atrophy or increased T2 weighted signal in the hippocampal region on $\mathrm{MRI},{ }^{1-12}$ was also only found for patients in the AHS group. It is interesting to note that the incidence of MRI abnormalities in both of the groups is lower than that found in a previous study, ${ }^{1}$ which involved some of the same subjects. The difference between the two sets of results seems to be related to the reviewer's threshold for calling a signal abnormal. (In the present study, only the most definite cases were considered abnormal.) Also whereas all of the patients in the AS group had preoperative grand mal seizures, this was not true for about a third of the patients in the AHS group.

Also, the AHS group differed from the other two groups in the number and types of auras experienced preoperatively; patients with AHS were more likely to have an aura and to have multiple aura symptoms. The aura types that were most likely to be experienced by patients in the AHS group, and not by those without hippocampal sclerosis, were smell and taste sensations and fear. An association of smell and taste auras with mesial temporal sclerosis was also reported by Falconer and Taylor. ${ }^{5}$ The association between fear and hippocampal damage is somewhat surprising, however, given that an aura of fear has previously been associated with electrical activity in the amygdala. ${ }^{19} 20$ The reason that only a few subjects with AS reported fear as part of their aura is not clear, but the number of subjects in this group who reported any aura at all was small. The paucity of auras experienced by the AS group also conflicts with the previous finding of a link between amygdalar stimulation and experimental auras. ${ }^{21}$

When interpreted blind to the nature of the pathology, preoperative EEGs showed more widespread abnormalities in AS than AHS. In AHS, background slowing and interictal spikes were largely confined to one temporal lobe whereas in AS, there was considerable propagation to extratemporal regions as well 
as a tendency for independent interictal spike activity to occur elsewhere in the brain. Recorded seizures reflected the propensity for epileptiform activity to be more widespread in AS. Considering this tendency for extratemporal involvement of EEG abnormalities in AS, it is not surprising that postoperative seizure control is less likely to be achieved than in AHS. As postoperative EEGs were not routinely carried out, we could not determine whether the extratemporal epileptiform abnormalities persisted in the patients with AS. This question should be answered in the future when more postoperative EEGs are done. As there seemed to be some ambiguity about the ECoG findings, we have also altered our ECoG methodology so that two depth electrodes are now inserted, one anteriorly aiming for the amygdala and one posteriorly into the hippocampus to try to assess more accurately interictal spike activity during the surgical procedure.

Before surgery, the highest failure rate on neuropsychological memory measures (especially on tests of memory for geometric designs) was seen for patients with AHS. These results are consistent with previous reports of memory disorders in association with hippocampal sclerosis. ${ }^{16}$ After surgery, only the AS group showed a significant decrease in memory performance. It is postulated that the memory disturbance seen preoperatively in the AHS group and postoperatively in both groups is caused by damage to the hippocampal region. This hypothesis is consistent with the results of monkey studies, which have indicated that significant memory deficits result only if both the amygdala, hippocampus, and underlying cortices are damaged, and not when a lesion involves only the amygdala..$^{22}$

It is interesting to note, however, that not only did the memory performance of the AS group tend to worsen postoperatively, but that the memory scores of the AHS group showed some improvement. This interaction reached significance only for the per cent delayed recall of the Rey figure, but the pattern is evident in table 3 for several other measures. Postoperative improvements in cognitive functioning for patients with hippocampal sclerosis have also been reported by McMillan et al. ${ }^{18}$ For the present study, careful examination of the subset of patients tested at follow up yielded no evidence of differences between the groups in side of lesion distribution or seizure outcome. Instead, it is proposed that after operation, improvements tend to be seen because patients are generally experiencing fewer seizures and they are on lower amounts of anticonvulsant medications. For the AHS group, these factors probably resulted in the increases seen in memory functioning and general intellectual level. For the AS group, it seems likely that the relatively recent loss of a functionally intact hippocampus outweighed these other positive influences. Thus although full scale IQ was not affected significantly for this group, memory abilities actually decreased.
The results of primate lesion studies ${ }^{22}$ as well as the description of a patient who sustained bilateral damage limited to the $\mathrm{CA}_{1}$ hippocampal region, ${ }^{24}$ indicate that appreciable memory problems are also seen in conjunction with focal hippocampal lesions that spare the amygdala. Therefore, it might be expected that preoperative isolated damage to the hippocampus (sparing the amygdala) in patients with epilepsy would result in memory deficits. In our experience, however, this pattern of sclerosis is not found in epileptic patients with hippocampal sclerosis; in these patients, some degree of AS is always present. Neuropsychological data from patients with focal hippocampal structural lesions, such as tumours, might be useful in exploring further the relative hippocampal and amygdalar contributions to memory.

The mechanism by which AS develops in the absence of hippocampal sclerosis is not known. It is unlikely that AS merely represents a mild form of mesial temporal sclerosis because, if that were the case, a better postoperative seizure outcome would be expected. Also, the factors that predispose a person to more extensive mesial temporal sclerosis, such as early childhood convulsions, are not present. Thus, AS may reflect a different pathogenesis of partial seizures than occurs in mesial temporal sclerosis, possibly one that involves a wider area of limbic and neocortex as suggested by the EEG findings and less successful results after temporal lobectomy.

We thank Ms Sofia Correia, Ms Maria Finmore, and Mr Mark Meninger for their help with data entry. We are grateful to $D$ Donald Lee for neuroradiological advice. To Drs W Blume, J Girvin and $\mathrm{H}$ Reichman we extend our thanks for the opportunity to study their patients. We also acknowledge $\mathrm{Dr} J$
McGlone, who collected some of the neuropsychological data.

1 Hudson LP, Munoz DG, Miller L, McLachlan RS, Girvin JP, Blume WT. Amygdaloid sclerosis in temporal lobe epilepsy. Ann Neurol 1993;33:622-31.

2 Falconer MA, Serafetinides EA, Corsellis JAN. Etiology and pathogenesis of temporal lobe epilepsy. Arch Neurol and pathogenesis

3 Duncan JS, Sagar HJ. Seizure characteristics, pathology, and outcome after temporal lobectomy. Neurology 1987;37:405-9.

4 Margerison JH, Corsellis JAN. Epilepsy and the temporal lobes: a clinical, electroencephalographic and neuropathological study of the brain in epilepsy, with particula reference to the temporal lobes. Brain 1966;89:499-530.

5 Falconer MA, Taylor DC. Surgical treatment of drug resistant epilepsy due to mesial temporal sclerosis. Arch Neurol 1968;19:353-61.

6 Miller LA, Munoz DG, Finmore M. Hippocampal sclerosi and human memory. Arch Neurol 1993;50:391-4.

7 Kuzniecky R, de la Sayette V, Ethier R, Melanson D, Andermann F, Berkovic S, et al. Magnetic resonance imaging in temporal lobe epilepsy: pathological correlations. Ann Neurol 1987;22:341-7.

8 Matsuda K, Yagi K, Mihara T, Tottori T, Watanabe Y, Seino M. MRI lesion and epileptogenic focus in temporal Seino M. MRI lesion and epileptogenic focus in tempora

9 Gates JR, Cruz-Rodriguez R. Mesial temporal sclerosis: pathogenesis, diagnosis, and management. Epilepsia pathogenesis, diagnosis,

10 Jackson GD, Berkovic SF, Tress BM, Kalnins RM Fabinyi GCA, Bladin PF. Hippocampal sclerosis can be reliably detected by magnetic resonance imaging. Neurology 1990;40:1869-75.

11 Berkovic SF, Andermann F, Olivier A, Ethier R, Melanson $\mathrm{D}$, Robitaille $\mathrm{Y}$, et al. Hippocampal sclerosis in tempora lobe epilepsy demonstrated by magnetic resonance imaging. Ann Neurol 1991;29:175-82.

12 Bronen RA, Cheung G, Charles JT, Kim JH, Spencer DD Spencer SS, et al. Imaging findings in hippocampa sclerosis: correlation with pathology. Am $\mathcal{f}$ Neuroradio 1991;12:933-40. 
13 Falconer MA, Cavanagh JB. Clinico-pathological considerations of temporal lobe epilepsy due to small focal lesions: a study of cases submitted to operation. Brain 1959;82:483-504.

14 VanBuren JM. The abdominal aura: a study of abdominal sensations occurring in epilepsy and produced by depth stimulator. Electroencephalogr Clin Neurophysiol 1963;15: stimulat

15 Feindel $W$, Rasmussen $T$. Temporal lobectomy with amygdalectomy and minimal hippocampal resection review of 100 cases. Can $\mathcal{F}$ Neurol Sci 1991;18:603-5.

16 Leonard G. Temporal lobe surgery for epilepsy: neuropsychological variables rela
Neurol Sci 1991;18:593-7.

17 Sagar HJ, Oxbury JM. Hippocampal neuron loss in temporal lobe epilepsy: correlation with early childhood convulsions. Ann Neurol 1987;22:334-40.

18 McMillan TM, Powell GE, Janota I, Polkey CE Relationships between neuropathology and cognitive functioning in temporal lobectomy patients. $\mathcal{f}$ Neurol Neurosurg Psychiatry 1987;50:167-76.

19 Gloor P. Temporal lobe epilepsy: its possible contribution to the understanding of the functional significance of the amygdala and of its interaction with neocorticaltemporal mechanisms. In: Eleftheriou BE, ed. The neurobiology of the amygadala. New York: Plenum, 1972: 423-57.

20 McLachlan RS, Blume, WT. Isolated fear in complex partial status epilepticus. Ann Neurol 1980;8:640-1.

21 Gloor P, Olivier A, Quesney LF, Andermann F, Horowitz $S$. The role of the limbic system in experiental phenomena of temporal lobe epilepsy. Ann Neurol 1982;12: ena of $129-44$.

22 Squire LR, Zola-Morgan S. Memory: brain systems and behaviour. TINS 1988;11:170-5.

23 Zola-Morgan S, Squire LR, Amaral DG. Lesions of the amygdala that spare adjacent cortical regions do not impair memory or exacerbate the impairment following lesions of the hippocampal formation. $\mathcal{F}$ Neurosi 1989 ; 9:1922-36.

24 Zola-Morgan S, Squire LR, Amaral DG. Human amnesia and the medial temporal region: enduring memory impairment following a bilateral lesion limited to field CAl of the hippocampus. $\mathcal{F}$ Neurosci 1986;6:2950-67. 